\title{
Werner Plumpe, André Steiner, Der Mythos von der postindustriellen Welt: Wirtschaftlicher Strukturwandel in Deutschland 1960 bis 1990
}

\section{Alain Alcouffe}

\section{OpenEdition}

\section{Journals}

Édition électronique

URL : http://journals.openedition.org/ifha/8545

DOI : $10.4000 /$ ifha. 8545

ISSN : 2198-8943

Éditeur

IFRA - Institut franco-allemand (sciences historiques et sociales)

Référence électronique

Alain Alcouffe, « Werner Plumpe, André Steiner, Der Mythos von der postindustriellen Welt: Wirtschaftlicher Strukturwandel in Deutschland 1960 bis $1990 »$, Revue de l'IFHA [En ligne], Date de recension, mis en ligne le 01 février 2017, consulté le 24 septembre 2020. URL : http:// journals.openedition.org/ifha/8545; DOI : https://doi.org/10.4000/ifha.8545

Ce document a été généré automatiquement le 24 septembre 2020.

(C)IFHA 
Werner Plumpe, André Steiner, Der Mythos von der postindustriellen Welt: Wirtschaftlicher Strukturwandel in Deutschland 1960 bis 1990

Alain Alcouffe

\section{RÉFÉRENCE}

Werner Plumpe, André Steiner, Der Mythos von der postindustriellen Welt: Wirtschaftlicher Strukturwandel in Deutschland 1960 bis 1990, Göttingen: Wallstein Verlag, 2016, 277 p., $29,90 €$ 
Le titre de cet ouvrage («le mythe du monde postindustriel: la transformation de la structure économique de l'Allemagne 1960-1990») renseigne moins sur le contenu de ce livre qu'il ne dévoile l'attachement si particulier de l'Allemagne pour son industrie. Aussi convient-il de détailler le contenu des cinq contributions qui le composent.

Dans une brève introduction (p. 7-14), les éditeurs, Werner Plumpe (université de Francfort) et André Steiner (université de Postdam), présentent différentes thèses intéressantes sur le traitement des sources dans les sciences sociales, les diagnostics de la situation contemporaine et les changements structurels. Suivent quatre contributions assez substantielles : une présentation générale et théorique des thèmes développés dans le livre et

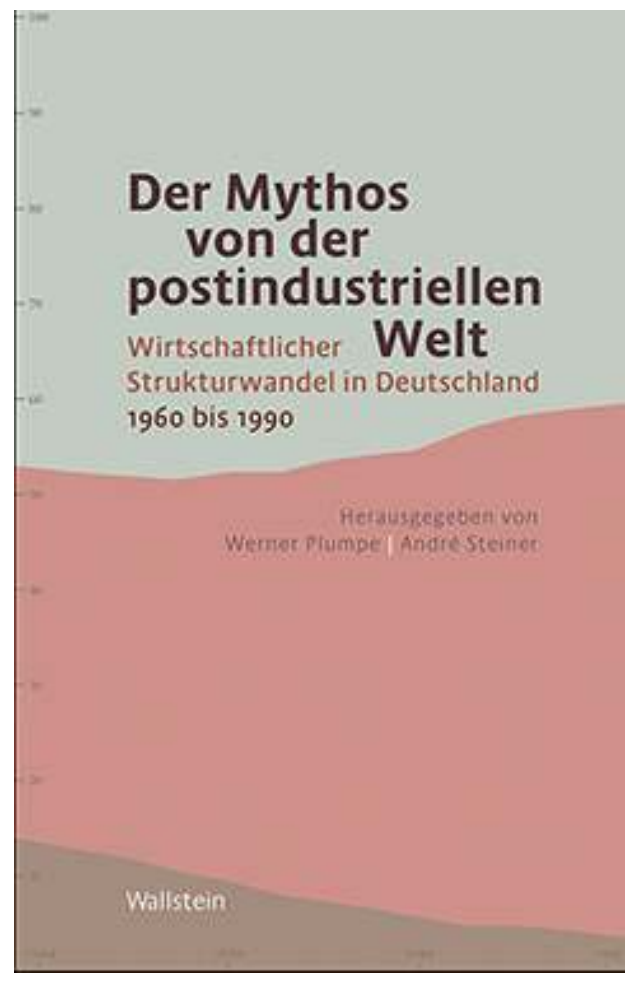
trois monographies sur différentes industries. Le choix des secteurs étudiés est très pertinent du fait de leur représentativité, allant de l'industrie classique (construction mécanique) au secteur du tourisme, en passant par l'industrie de la musique enregistrée.

Dans la première contribution intitulée «Adieu à l'industrie?» (p. 15-54), A. Steiner entend retracer à grands traits « la transformation des structures économiques dans l'Allemagne de l'Ouest et de l'Est depuis les années 1960 ». Toutes deux ont connu les mêmes tendances sectorielles, en particulier le recul du secteur primaire au profit du secteur tertiaire, même si ce recul a pu être décalé dans le temps et d'ampleur différente. L'auteur voit dans ces changements moins un déplacement de la frontière entre les secteurs qu'une nouvelle interpénétration entre les structures productives de l'industrie et des services. Du fait des nouvelles technologies, le contenu en services s'accroît dans la production industrielle, de sorte que l'on voit apparaître « un domaine hybride entre l'industrie au sens traditionnel du terme et ce que l'on classe communément dans le secteur des services » (p. 28). Cette grille d'analyse fait surgir des différences et des similitudes importantes entre les deux Allemagnes. A. Steiner soutient en effet que les secteurs industriels et des services ont été inextricablement liés des deux côtés du rideau de fer, et qu'il n'est donc guère possible de parler de désindustrialisation (p. 31). RDA et RFA ne diffèrent que par la nature des industries qui ont relevé avec succès les défis du changement structurel, comme le montre A. Steiner à travers les « activités des acteurs de changement structurel » (p. 34). Il met l'accent sur les stratégies des acteurs (entrepreneurs ou collectifs) dans les trois monographies sectorielles. La conclusion de cette contribution aborde la période après 1990 durant laquelle s'est poursuivie l'intégration étroite de l'industrie et des services dans l'Allemagne réunifiée. Elle permet à l'auteur de critiquer la périodisation proposée par Anselm Doering-Manteuffel et Lutz Raphael dans leur livre publié en 2010, Nach dem Boom: Perspektiven auf die Zeitgeschichte seit 1970 (Gottingen: Vandenhoeck \& Ruprecht) 
qui défend au contraire l'idée d'une césure ou d'une multiplicité de ruptures après le boom des années 1960-1980. Au lieu de parler comme eux d'une rupture, il préfère décrire le changement structurel comme un processus continu s'intensifiant par phases (p. 53).

La première des trois études sectorielles est intitulée «Une vieille industrie face à de nouveaux défis : essors et déclins de la construction de machines dans l'Allemagne de l'Est et de l'Ouest depuis les années $1960 »$ (p.55-119). Ralf Ahrens y opère une distinction entre «vieille industrie» et «nouvelle industrie à forte intensité de connaissances »: «Au cœur de la distinction se trouvent les réactions aux nouvelles conditions de la concurrence depuis les années $1960 »$ (p. 56). L'auteur se concentre sur les conditions structurelles et sur les évolutions spécifiques à l'industrie, de même que sur les champs d'action des acteurs concernés. Parmi les premières, R. Ahrens s'intéresse, entre autres, à l'appréciation du Deutsche Mark en Allemagne de l'Ouest ainsi qu'au "problème de l'innovation propre à l'industrie » (p.65). Pour la RDA, il détaille "diverses faiblesses en matière d'efficience et d'investissement» (p. 94). Malgré ces tendances et ces conditions plutôt négatives, l'industrie de la fabrication de machines a relevé « les défis du changement structurel tant à l'Est qu'à l'Ouest mieux que d'autres industries traditionnelles" (p.114). L'auteur confirme l'observation macroéconomique d'une tertiarisation croissante en réponse aux changements structurels. Mais il s'agit surtout d'un changement à l'intérieur du secteur à la suite du renforcement du secteur « recherche et développement »; l'évolution n'a pas modifié l'image d'une branche orientée vers l'innovation et à vocation technique (p.118). R. Ahrens montre que les réponses au changement structurel apportées en RDA sont passées par la nationalisation et la formation de combinats. Ceux-ci tendaient à développer la recherche et le développement (p. 105), mais cela n'a pas conduit au même niveau de tertiarisation qu'en RFA.

Christian A. Müller étudie l'industrie de la musique enregistrée dans sa contribution intitulée "Dévoiler l'arrière-plan non créatif: l'industrie phonographique dans la transformation structurelle de la RDA et de la RFA des années 1950 aux années 1980 » (p. 120-172). Il s'agit d'un secteur dont l'importance économique est faible mais qui joue un rôle important dans l'évolution culturelle de la société et qui se situe dans le champ intermédiaire entre industrie et services évoqué par A. Steiner. Dans son introduction envisageant «les marchés esthétiques comme défi structurel de l'industrie de la musique enregistrée dans l'Allemagne de l'Est et de l'Ouest », C. Müller se place dans le cadre d'analyse des marchés esthétiques définis par le sociologue suédois Patrik Aspers comme des marchés structurés par des valeurs esthétiques (voir son ouvrage Markte, Wiesbaden: Springer VS, 2015). Contrairement aux études culturelles et sociohistoriques "de la musique enregistrée en tant que produit de consommation" (p.120), C. Müller s'intéresse au domaine jusque-là négligé de l'offre de musique enregistrée (p. 121). Il distingue quatre phases pour lesquelles il met en évidence les particularités et les similitudes entre l'industrie à l'Est et à l'Ouest. Les similitudes concernent, entre autres, un «transfert et un échange de répertoire artistique » assez important (p. 168) à partir des années 1950. De plus, les industries ont été confrontées à des problèmes similaires : comment trouver des produits correspondant aux attentes des consommateurs? Comment optimiser la production et la distribution? C. Müller arrive à la conclusion qu'il n'est plus possible de distinguer un secteur industriel du disque et un secteur de service correspondant à la mise sur le marché (p.172). Il 
propose de substituer à cette distinction l'étude du changement structurel et de ses effets « du point de vue de la chaîne de valeur spécifique » (p. 170).

Jörg Lesczenski consacre la dernière contribution à l'industrie du tourisme sous le titre "Vacances en rayon: les voyagistes et la transformation du tourisme 'tout compris' dans les deux États allemands (1960-1990)»(p.173-257). Dans son introduction, l'auteur recense les études parues sur les évolutions du tourisme et indique que ses propres recherches s'appuient sur le dépouillement des principaux acteurs du secteur tant à l'Ouest qu'à l'Est. Il s'intéresse ensuite au développement du tourisme organisé des années 1961-1962 aux années 1968-1973 sous la poussée d'une demande croissante et la pression des coûts. Il constate que «la clientèle est devenue plus différenciée, sophistiquée, riche, ouverte au monde». En raison de la concurrence entre les multiples opérateurs, la pression sur les coûts s'est intensifiée et les opérateurs ont réagi en rationalisant leur offre et en développant des études de marché. La principale différence entre les deux États se serait produite dans les années 1970, quand a commencé, en RFA, une «transformation rapide des structures du tourisme de masse, portée par le libre jeu des acteurs du marché », tandis qu'à l'Est « le développement du tourisme organisé [...] était plus que jamais soumis à l'intervention du gouvernement " (p. 214). J. Lesczenski conclut qu'en ce qui concerne la transformation des structures de l'industrie du tourisme dans les années 1970, on ne peut pas parler de crise «profonde, d'adaptation et de transformation » (p. 254 et suivantes), comme Morten Reitmayer et Ruth Rosenberger le soutiennent pour « les entreprises du secteur secondaire » (p. 255) (Voir Morten Reitmayer, Unternehmen am Ende des "goldenen Zeitalters »: die 1970er Jahre in unternehmens- und wirtschaftshistorischer Perspektive, Essen: Klartext, 2008). On peut au contraire parler d'« une phase d'adaptation accélérée » (ibid.).

Le livre a fait l'objet de recensions élogieuses en Allemagne (Voir Jörn Eiben, H-Net Reviews, avril 2016 et Andrea Gabler dans Sozial.Geschichte Online, Heft 19/2016), en dehors du choix du titre. Pour un lecteur français, l'approche consistant en un traitement synchronique et diachronique des deux États allemands est très intéressante dans la mesure où elle fait apparaître des convergences inattendues audelà des différences entre les acteurs et les structures étatiques à l'Est et à l'Ouest. Pour un économiste travaillant sur les débats actuels autour de la stagnation séculaire, l'ouvrage se livre aussi à une remise en cause salutaire de la théorie des trois secteurs (primaire, secondaire et tertiaire), même si on peut trouver le traitement de Fourastié un peu superficiel. La critique de la thèse d'une société post-industrielle liée à un effacement des frontières entre industrie et services, si elle traduit un attachement profond en Allemagne à la civilisation industrielle, ne manquera pas de susciter des discussions et appelle à des comparaisons avec d'autres pays.

\section{INDEX}

Index chronologique : Époque contemporaine

Thèmes : Histoire économique 


\section{AUTEURS}

\section{ALAIN ALCOUFFE}

Université Toulouse Capitole 